\title{
Linac Design Study for an Intense Neutron-Source Driver*
}

\author{
M.T. Lynch, A. Browman, R.DeHaven, R. Jameson, A. Jason, G. Neuschaefer, P. Tallerico, and A. Regan \\ Los Alamos National Laboratory \\ Los Alamos, NM 87545
}

*Supported by the US Department of Energy, Office of Defense Programs

\section{Abstract}

The 1-MW spallation-neutron source under design study at Los Alamos is driven by a linac-compressor-ring scheme that utilizes a large portion of the existing Los Alamos Meson Physics Facility (LAMPF) linac, as well as the facility infrastructure. The project is referred to as the National Center for Neutron Research (NCNR). A second phase of the proposal will upgrade the driver power to $5 \mathrm{MW}$. A description of the 1-MW scheme is given in this paper. In addition, the upgrade path to the substantial increase of beam power required for the $5 \mathrm{MW}$ scenario is discussed.

\section{Introduction}

A proposal is being developed to modify LAMPF for use as a spallation-neutron source ${ }^{1}$. The spallation source would require a $1 \mathrm{MW}$ average beam as the input driver, with consideration being given for a future upgrade to 5MW. The 1-MW driver would require that the LAMPF facility consistently and reliably provide $30 \mathrm{~mA}$ peak, $1.25 \mathrm{~mA}$ average current at $800 \mathrm{MeV}$. A large portion of the LAMPF accelerator can remain intact, but the front end of the accelerator would need replacement to be suitable for this application. This paper addresses the issues associated with the LAMPF linac and RF systems. Other papers at this conference address other parts of the spallation source ${ }^{1,2}$.

\section{The LAMPF Accelerator}

The existing LAMPF accelerator consists of dual Cockraft-Walton injectors $\left(\mathrm{H}^{+}\right.$and $\left.\mathrm{H}^{-}\right)$with an output energy of $750 \mathrm{keV}$. The current is chopped at a 201.25 MHz rate and then is accelerated to $100 \mathrm{McV}$ in a 201.25 MHz Drift Tube Linac (DTL). A side-coupled linac structure, operating at $805 \mathrm{MHz}$, accelerates the beam to the final energy of $800 \mathrm{MeV}$. A line diagram of the LAMPF accelerator is shown in Figure 1. Details of the LAMPF accelerator operation are listed in Table 1.

\begin{tabular}{|lc|}
\hline \multicolumn{2}{|c|}{ Table 1} \\
LAMPF Accelerator Operating Parameters \\
\\
Output Energy & $800 \mathrm{MeV}$ \\
Macropulse Rep Rate & $120 \mathrm{~Hz}$ \\
Pulse Width & $1 \mathrm{~ms}$ \\
Micropulse Rep Rate & $201.25 \mathrm{MHz}$ \\
& \\
Production Capability: & $17 \mathrm{~mA}, \mathrm{H}^{+}$ \\
Iavg during macropulse & $10 \%$ \\
Duty Factor & $1.4 \mathrm{MW}$ \\
Avg. Beam Power & \\
& \\
Experimental Operations: & $21 \mathrm{~mA}$ \\
Iavg during macropulse & $\mathrm{H}^{+}$; and $7 \mathrm{~mA}^{-}$) \\
\hline
\end{tabular}

The baseline design for the neutron source driver completely replaces the Cockroft-Walton injectors and the 201.25 MHz DTL. This section of the accelerator has been the most unreliable part of the accelerator in the recent past. The $805 \mathrm{MHz}$ SCL would remain essentially intact.

\section{Linac for the Intense Neutron Source}

The linac for the proposed spallation source would use as

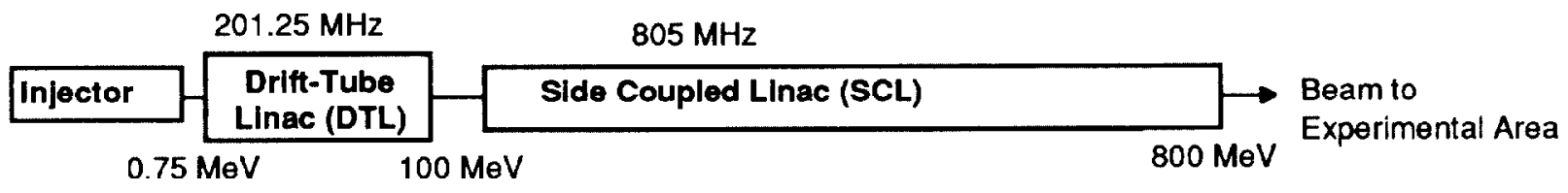

Figure 1. Line Diagram of the exisiting LAMPF Accelerator 
much of the existing LAMPF accelerator as possible. Refer to Figure 2. The front end of LAMPF would be replaced with a $75 \mathrm{keV} \mathrm{H}^{-}$injector, a $402.5 \mathrm{MHz} \mathrm{RFQ}$ and DTL to get the beam to $20 \mathrm{MeV}$, and a second DTL at $805 \mathrm{MHz}$ to raise the energy to $100 \mathrm{MeV}$. The existing SCL would remain to accelerate the beam to 800 $\mathrm{MeV}$ for insertion into the storage rings 1 . The RFQ has a relatively high output energy $(\sim 7 \mathrm{MeV})$. The DTL structures are all standard Alvarez DTL tanks. This front end uses relatively high gradients in order to fit into the existing LAMPF tunnel. The primary drawback to the use of high gradients is increased copper losses. The front end therefore operates at a lower efficiency (beam output power divided by RF power in) compared to an ideal structure with unlimited real estate.

\section{Beam Macropulse Structure and Repetition Rate}

The storage ring design for this neutron spallation source has a one-time transit time of $671 \mathrm{~ns}$. In order to allow time for kicker magnet rise-time, the beam must have a notch, so the beam time profile is $436 \mathrm{~ns}$ "on" and 235 ns "off". In order to obtain an average beam power of 1 $\mathrm{MW}$, the average beam stored in the rings must be 1.25 $\mathrm{mA}$ (at $800 \mathrm{MeV}$ ). Assuming a $90 \%$ capture efficiency in the rings, the linac average output current must be 1.39 $\mathrm{mA}$. Assuming a rep rate of $60 \mathrm{~Hz}$ and a macropulse length of $1.2 \mathrm{~ms}$, the average current during the macropulse must be $19.5 \mathrm{~mA}$. Given the chopping rate described above $(65 \%)$, the peak current during the "on" time of the macropulse is $30 \mathrm{~mA}$.

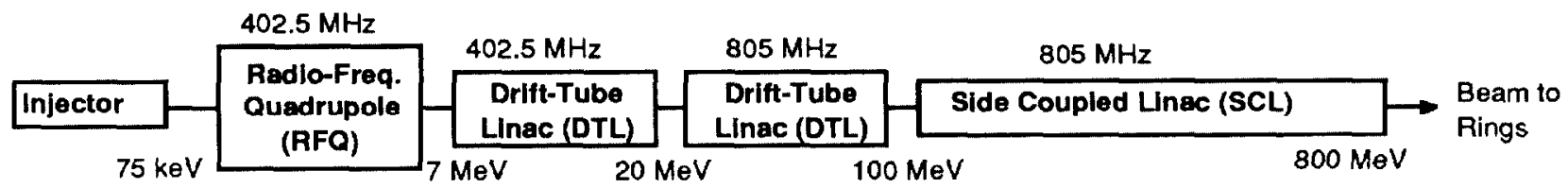

Figure 2. Line Diagram of the Proposed NCNR Driver Linac.

The SCL consists of 44 modules, each driven by a 1.25 MW klystron. Very little of this portion of the accelcrator will need to be changed for the $1 \mathrm{MW}$ driver. The primary changes would come in the low-level feedback/feedforward controls.

Structure power, beam power, and RF power requirements for the RFQ, DTL's, and SCL are shown in Table 2. The RF generator size for the new sections is nominally $1.25 \mathrm{MW}$ based on the existing Ground Test Accelerator (GTA), LAMPF, and Ballistic Missile Early Warning System (BMEWS) klystron technology. Twoklystron modulators based on the GTA technology 3 would be used for all new klystrons. Small RF generators ( $10 \mathrm{~kW}$ or less) are needed for matching section cavities between the RFQ and first DTL and between the two DTL's. These amplifiers will either be solid state (if the power is low) or triode or tetrode-based cavity amplifiers (if more than a few $\mathrm{kW}$ is needed).
The output beam is shared between two storage rings 1 , one operating at $40 \mathrm{~Hz}$ and one at $20 \mathrm{~Hz}$. This output requires that the injector be pulsed at a $120 \mathrm{~Hz}$ rate, but the RF in the accelerator cavities is pulsed only at the "syncopated" rate shown in Figure 3. The RF therefore must be capable of repeating at a $120 \mathrm{~Hz}$ rate but averages only 60 pulses per second.

\section{The LAMPF SCL}

There is one area of concern with the SCL. Some of the low energy modules are very long because four separate tanks are bridge-coupled together and driven by a single klystron. The measured group delay from the drive point to one end of the structure is about $1-\mu$ s in these modules, and the loaded $Q$ of the structure is about 9000 , giving a fill time of about $3.5 \mu \mathrm{s}$. It is therefore impossible for the RF feedback system to keep up with the chopping rate of the beam because the response time

Table 2

Sections of NCNR and Power Required

\begin{tabular}{|c|c|c|c|c|c|c|c|}
\hline Section & $\begin{array}{l}\text { Frequency } \\
(\mathrm{MHz})\end{array}$ & \# of Tanks & $\begin{array}{l}\text { \# of RF } \\
\text { Modules }\end{array}$ & $\begin{array}{l}\text { Beam Power } \\
(\mathrm{MW})\end{array}$ & $\begin{array}{l}\text { Structure } \\
\text { Power (MW) }\end{array}$ & $\begin{array}{l}\text { Total RF } \\
\text { Power* (MW) }^{*} \text {. }\end{array}$ & New? \\
\hline$\overline{\mathrm{RFQ}}$ & 402.5 & 1 & 2 & 0.14 & 1.4 & 1.93 & yes \\
\hline DTL-1 & 402.5 & 2 & 2 & 0.25 & 1.23 & 1.85 & yes \\
\hline DTL-2 & 805 & 15 & 8 & 1.6 & 4.2 & 7.25 & yes \\
\hline $\mathrm{SCL}$ & 805 & 104 & 44 & 13.7 & 27 & 51 & no \\
\hline
\end{tabular}

*Total RF Power includes a $25 \%$ margin for control and losses 
is so much slower than the chopping rate. Since the beam chopping pattern is repetitive and known, one could consider the use of feedforward and a large amount of RF overdrive to correct the beam chopping transients. However the long group delay of the structure precludes successful control. The RF cannot get from the drive point to the structure ends fast enough to react to the beam.

\section{Plans for Upgrade to $5 \mathrm{MW}$}

The plans for upgrade to $5 \mathrm{MW}$ are very sketchy at this point. The baseline accelerator for the $5 \mathrm{MW}$ source has an output energy of $1.6 \mathrm{GeV}$ and a current level of 75 $\mathrm{mA}$. The pulse width and duty factor are unchanged. This accelerator would almost certainly require funneling at the

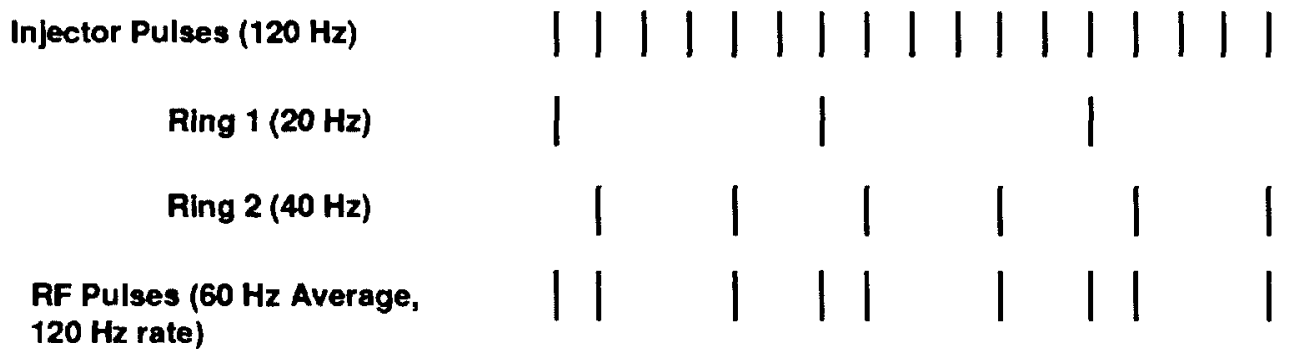

\section{Figure 3. Distribution of pulses to the two rings for NCNR}

Two items can ameliorate the beam loading problem due to a chopped beam. If the structure has enough stored energy, there will be a minimal drop in the structure field from the front to the rear of the pulse. Initial analysis of this variation in fields with NCNR currents shows a droop of $1.5 \%$ in one module during the "on" time of the beam. This is being investigated further to see if the beam transport system will carry the beam with this amount of energy variation.

Another solution being considered is to break the modules in half, and drive each half independently. Not all modules are large, so this would only be done on a few of the SCL modules. The RF system would still not be fast enough to respond at the chopping rate, but the droop per tank would be cut by a factor of 4 (droop is proportional to $\mathrm{N}^{2}, \mathrm{~N}=$ number of cells).

An experiment is planned for early June, 1993, in which two different modules of the LAMPF accelerator will be operated with GTA style feedback controls. NCNR will require that LAMPF operate at what has historically been the limits of its capability. One of the problems has been beam spill at high operating currents. The major portion of the lost beam at LAMPF occurs during the beam turnon transient. The GTA-style feedback controls allow precise setting of the feedback gains and have the capability of adaptive feedforward control ${ }^{4}$. The adaptive feedforward technique is an extremely powerful tool for the correction of repetitive, systematic errors. For more detail about the use and applications of adaptive feedforward refer to the paper by Ziomek ${ }^{5}$, et al, in these proceedings. low energy end (between the two DTL's). The path to 1.6 $\mathrm{GeV}$ can go two ways. In the first, an afterburner (perhaps superconducting) would be added to the existing structure to raise the output energy from $800 \mathrm{MeV}$ to $1600 \mathrm{MeV}$. This scheme assumes that operation at 1 MW has shown the capability for successful operation with 2.5 times more current. In the second scheme, the complete SCL structure and perhaps some of the DTL structures would be replaced with a new structure (perhaps superconducting).

\section{References}

[1] A. Jason, et al, "A Los Alamos Design Study for a High-Power Spallation-Neutron-Source Driver," these proceedings.

[2] B. Blind, et al, "Lattice Design of the LANL Spallation-Source Compression Rings," these proceedings.

[3] P.J. Tallerico, et al, "Progress on the GTA 850-MHz RF System," LANL report number LA-CP-90-231, Proceedings of the NPB Technical Interchange Symposium, May 21-24, 1990.

[4] S. Jachim, et al, "The Los Alamos VXI-Based Modular RF Control System," these proceedings.

[5] C. Ziomek, et al, "Results of Adaptive Feedforward on GTA," these proceedings. 